Original paper

\title{
Passive marker tracking via phase-only cross correlation (POCC) for MR-guided needle interventions: Initial in vivo experience
}

\author{
Axel Joachim Krafft ${ }^{\mathrm{a}, *, 1}$, Patrik Zamecnik ${ }^{\mathrm{b}, 1}$, Florian Maier ${ }^{\mathrm{a}}$, André de Oliveira ${ }^{\mathrm{c}}$, \\ Peter Hallscheidt ${ }^{\mathrm{d}}$, Heinz-Peter Schlemmer ${ }^{\mathrm{b}}$, Michael Bock ${ }^{\mathrm{a}, \mathrm{e}}$ \\ ${ }^{a}$ Department of Medical Physics in Radiology, German Cancer Research Center, Heidelberg, Germany \\ ${ }^{\mathrm{b}}$ Department of Radiology, German Cancer Research Center, Heidelberg, Germany \\ ${ }^{\mathrm{c}}$ Siemens Healthcare, Erlangen, Germany \\ ${ }^{\mathrm{d}}$ Department of Diagnostic Radiology, University of Heidelberg, Heidelberg, Germany \\ ${ }^{\mathrm{e}}$ Department of Radiology, Medical Physics, University Hospital Freiburg, Germany
}

\section{A R T I C L E I N F O}

\section{Article history:}

Received 30 April 2012

Received in revised form 7 September 2012

Accepted 20 September 2012

Available online 17 October 2012

\section{Keywords:}

Interventional magnetic resonance imaging

MR-guided interventional procedures

MR-guided prostate biopsy

MR-guided needle intervention

Interventional radiology

\begin{abstract}
A B S T R A C T
Purpose: In this work, a passive tracking sequence employing a phase-only cross correlation (POCC) algorithm was studied with a focus on the in vivo applicability of the technique. Therefore, MR-guided needle interventions were performed in a phantom and two animal experiments.

Methods: The targeting accuracy was quantified in an agarose phantom with 15 fiducials. For each fiducial, the distance between needle trajectory and target point was measured. In a first animal experiment at $3 \mathrm{~T}$, the prostate of a pig was punctured under POCC guidance. Second, POCC-based tracking was performed during a laser-induced thermal therapy procedure in peripheral porcine muscle tissue at $1.5 \mathrm{~T}$.

Results: In the phantom experiment, the 15 fiducials were penetrated with a mean accuracy of $1.5 \pm 0.9 \mathrm{~mm}$ (mean duration for one puncture about $2 \mathrm{~min}$ ). In the first animal experiment, the center of the pig's right prostatic lobe was accurately punctured within $15 \mathrm{~min}$. In the second, targeting and insertion of the needle could be performed within $5 \mathrm{~min}$ and a thermal lesion was successfully created. Conclusion: Our initial experience with the POCC-based tracking sequence indicates that this technique has the potential as an accurate and versatile tool for in vivo MR-guided needle interventions.
\end{abstract}

() 2012 Associazione Italiana di Fisica Medica. Published by Elsevier Ltd. All rights reserved.

\section{Introduction}

In recent years MRI has been increasingly used in image-guided minimally invasive procedures $[1,2]$. Compared to CT or ultrasound, MRI combines an excellent soft tissue contrast without the need of ionizing radiation. For a precise localization of biopsy devices used for breast or prostate interventions, a high spatial resolution is needed which can lead to long MRI measurement times. Thus, durations of such interventions are often longer with MR guidance compared to CT or ultrasound guidance. Procedure times for MRguided biopsies have been reported between 40 and 70 min for the breast $[3,4]$ and in the range of $55 \mathrm{~min}$ up to $2 \mathrm{~h}$ for the prostate [5-8].

\footnotetext{
* Corresponding author. Current address: St. Jude Children's Research Hospital, Department of Radiological Sciences, 262 Danny Thomas Place, MS 220, Memphis, TN 38105, USA. Tel.: +1901595 7731; fax: +19015953981.

E-mail address: axel.krafft@stjude.org (A.J. Krafft).

${ }^{1}$ Equal contribution.
}

In MR-guided needle biopsies, markers are often employed to detect the orientation of the interventional device [6,9]. As these interventions require a high signal-to-noise ratio (SNR) for an adequate identification of target and device, they are preferably performed in closed-bore high field MR systems. Unfortunately, these systems only allow for a limited patient access. As there is no direct line of sight, optical markers cannot be used for device tracking. Passive MR markers have been shown to facilitate the interventional procedure. However, they are often localized in the MR images by aligning the scan plane manually with the marker. These re-alignments are time-consuming and can take up to minutes after each manipulation of the device. Furthermore, the geometric accuracy is limited since the operator has to visually assess whether the imaging slice is parallel to the planned needle trajectory.

Recently, a passive tracking approach based on a phase-only cross correlation (POCC) algorithm has been proposed [10,11] for an accurate and faster targeting procedure. The POCC algorithm is used to detect the position of a passive MR marker in real-time. The position and orientation information of the marker is employed to 
automatically align an imaging slice, so that the imaging plane follows the passive marker. Consequently, navigation of the device to the biopsy target is achieved similar to an ultrasound-guided procedure.

In this work, the accuracy of the POCC-based tracking for MRguided needle interventions was evaluated in a phantom experiment. Then, the technique was tested in two animal experiments with pigs - a puncture of a prostate and a laser-induced thermal therapy (LITT) in peripheral muscle tissue - at different field strengths ( $3 \mathrm{~T}$ and $1.5 \mathrm{~T}$ ) to study its applicability for MR-guided needle interventions.

\section{Methods}

\section{POCC tracking sequence}

The POCC tracking sequence [10] was developed to automatically follow a commercially available passive marker (Prostate Needle Guide, Invivo Germany, Schwerin, Germany) consisting of a plastic cylinder (length: $85 \mathrm{~mm}$, diameter: $12 \mathrm{~mm}$ ) filled with contrast agent solution (Magnevist ${ }^{\circledR} / \mathrm{H}_{2} \mathrm{O}$ : $1 / 100$, Bayer Schering Pharma AG, Berlin, Germany) and featuring a central hole (inner diameter: $3 \mathrm{~mm}$ ) through which the needle can be inserted. At the beginning of the procedure, the marker is manually pre-positioned near the targeted region with the cylinder's symmetry axis (reflecting the theoretical trajectory of the needle) pointing at the expected target location. For example, for prostate biopsies the marker would be placed inside of the rectum [6], and for peripheral percutaneous interventions the marker would be positioned above the skin near the expected entry point of the needle.

Initially, two parallel tracking slices $\left(\mathrm{T}_{1}\right.$-weighted FLASH images, distance: $40 \mathrm{~mm}$ ) are manually positioned approximately perpendicular to the cylinder's symmetry axis. In these tracking images the passive marker appears as a ring-like structure. The positions of these structures are calculated by applying a POCC algorithm [10,11]. A detailed description of the POCC tracking algorithm can be found in [10]. In short, the POCC algorithm obtains the position $P(x, y)$ of an object as the maximum from a normalized convolution of a current MR image $I(x, y)$ and a synthetic image $M(x, y)$ of the object containing the object at its center:

$P(x, y)=\max [\operatorname{POCC}(x, y)]=\max \left[\frac{I(x, y)}{\|I(x, y)\|} * \frac{M(x, y)}{\|M(x, y)\|}\right]$

The synthetic image $M(x, y)$ is generated according to the physical dimensions of the object, i.e. inner and outer diameter of the passive marker, and the relevant MR sequence parameters, i.e. field of view (FOV) and matrix size. Instead of a time-consuming convolution, Eq. (1) is efficiently processed as a multiplication using the k-space representations of $I$ and $M$. Finally, a sub pixel refinement of the marker position as estimated from POCC is achieved using a centerof-mass algorithm $[10,12]$. The calculated position information is then employed to automatically align a subsequent targeting image parallel to the cylinder's symmetry axis. The targeting image is acquired with a balanced steady-state free precision sequence (bSSFP) to provide a $\mathrm{T}_{2}$-dominated contrast.

The entire cycle of interleaved acquisitions of tracking and targeting images runs continuously, so that the targeting slice follows the position of the marker. The marker is manually moved until the cylinder's symmetry axis is pointing at the pre-defined target. To facilitate image guidance during the targeting process, the theoretical needle axis is calculated based on the marker positions and overlaid onto the current bSSFP targeting image. These images are presented to the interventional radiologist via the system's in-room monitor inside of the RF cabin. In this study, implementations of the POCC sequence for clinical whole body MR systems with field strengths of $3 \mathrm{~T}$ and $1.5 \mathrm{~T}$ (MAGNETOM TimTrio and Symphony, Siemens Healthcare, Erlangen, Germany) were used. Depending on the specific imaging parameters, the total duration $\left(\mathrm{TA}_{\mathrm{tot}}\right)$ of one imaging cycle amounted to $1-2 \mathrm{~s}$.

\section{Accuracy evaluation in phantom at $3 \mathrm{~T}$}

Although the accuracy of the POCC tracking has been assessed in a prototype setup [10], a phantom experiment was performed to measure the targeting accuracy in a more realistic scenario with multiple targets. In Ref. [10] an accuracy of $1.5 \pm 1.1 \mathrm{~mm}$ for targeting of five ellipsoidal capsules (size: $5 \times 5 \times 10 \mathrm{~mm}^{3}$ ) placed under a thin layer of gelatin (depth: $5 \mathrm{~mm}$ ) was found. Here, the accuracy was measured for 15 fiducials (cranberries, mean diameter: $7.8 \pm 0.2 \mathrm{~mm}$ ) embedded in a non-transparent gel phantom (3\% agarose, doped with MR contrast agent Magnevist ${ }^{\circledR} / \mathrm{H}_{2} \mathrm{O}: 1 /$ 800, Bayer Schering Pharma AG, Berlin, Germany; Fig. 1a) at various depths ranging from $23 \mathrm{~mm}$ to $78 \mathrm{~mm}$ (mean depth: $72 \pm 24 \mathrm{~mm}$ ) to simulate typical target depths during transrectal prostate biopsies (typical target depth in ultrasound-guided prostate biopsies about $60-65 \mathrm{~mm}$ ). The phantom had a size of $120 \times 95 \times 60 \mathrm{~mm}^{3}$ (length $\times$ width $\times$ height) and was composed of four layers (mean thickness: $15 \mathrm{~mm}$, Fig. 1b) where each of the three lower layers contained five fiducials. For MRI signal reception the spine array coil and a flexible coil were used.
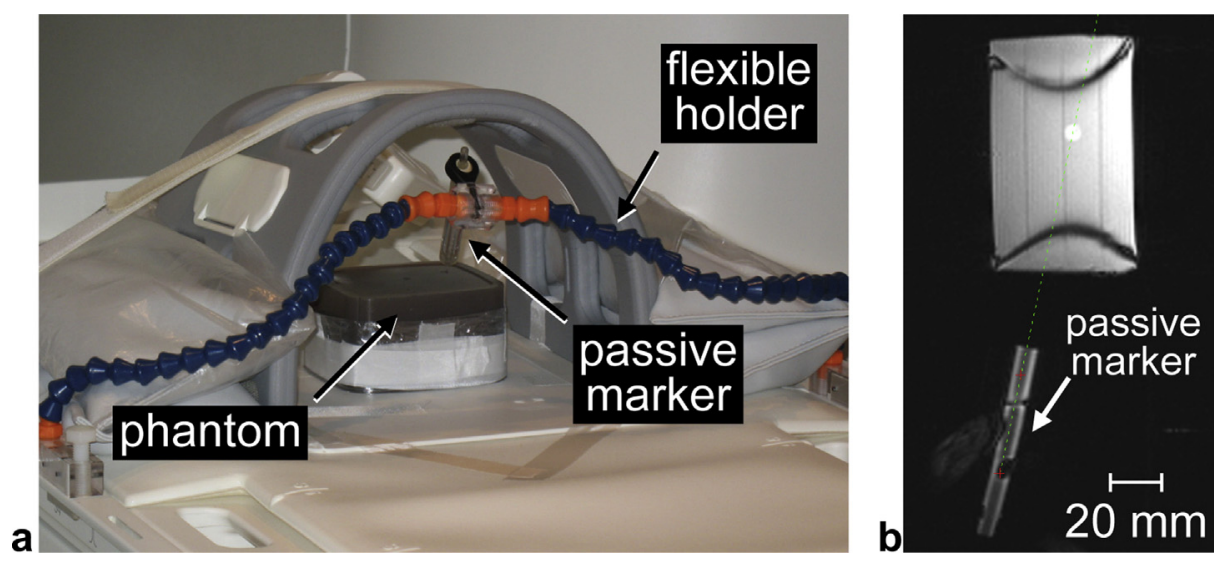

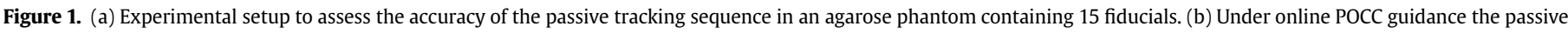
marker is manipulated (finger of the interventional radiologist visible) until the cylinder's symmetry axis (dotted line) is aligned with the center of the pre-defined target. 
At the beginning of the experiment, the passive marker was attached to a customized flexible holder (Fig. 1a) which was fixed on both sides of the patient table of the MR scanner. The holder's distal end featured a ball joint for manual manipulation of the marker. After the acquisition of a $\mathrm{T}_{2}$-weighted 3D turbo spin echo (TSE) roadmap data set, the following procedure was performed for each of the 15 fiducials: The passive marker was manually moved under guidance of the POCC tracking sequence (FLASH tracking slices: TR/TE $=4.0 / 1.9 \mathrm{~ms}$, FOV: $280 \times 280 \mathrm{~mm}^{2}$, matrix: $256 \times 256, \alpha_{\mathrm{FLASH}}=25^{\circ}$, thickness: $5.0 \mathrm{~mm}$, partial Fourier: $5 / 8$; bSSFP targeting slice: TR/TE $=4.0 / 1.9 \mathrm{~ms}$, FOV: $280 \times 280 \mathrm{~mm}^{2}$, matrix: $256 \times 256, \alpha_{\mathrm{bSSFP}}=70^{\circ}$, thickness: $5.0 \mathrm{~mm}$, partial Fourier: $5 / 8 ; \mathrm{TA}_{\mathrm{tot}}=1.9 \mathrm{~s}$ ) until the theoretical needle axis overlaid onto the targeting images was aligned with the center of the fiducial. Then, manipulation of the marker was stopped, and a customized MR-compatible puncture needle (length: $270 \mathrm{~mm}$, diameter: $12 \mathrm{G}$; Invivo $\mathrm{GmbH}$, Schwerin Germany) was manually advanced towards the target. The penetration of the needle was monitored with a standard bSSFP sequence without tracking (TR/ $\mathrm{TE}=4.0 / 1.7 \mathrm{~ms}$, FOV: $280 \times 280 \mathrm{~mm}^{2}$, matrix: $256 \times 256, \alpha=80^{\circ}$, thickness: $3.0 \mathrm{~mm}$, partial Fourier: 7/8). Therefore, position and orientation of these bSSFP images were copied from the last targeting image before marker manipulations were stopped. During needle insertion tracking was disabled, as the marker position was kept fixed by the holder and the needle artifact could lead to distortions of the ring-like marker signature causing incorrect marker tracking. After target penetration the needle was withdrawn leaving a small, clearly circumscribed air-filled channel (diameter about $1 \mathrm{~mm}$ ) in the semi-rigid agarose phantom. For each target the time needed for targeting and needle insertion was measured.

Finally, a high-resolution $\mathrm{T}_{1}$-weighted $3 \mathrm{D}$ data set ("post-intervention data set") was acquired (FLASH, 96 slices, TR/TE $=4.4 /$ $1.9 \mathrm{~ms}, \mathrm{FOV}=120 \times 150 \mathrm{~mm}^{2}$, matrix: $208 \times 256$, thickness: $1.0 \mathrm{~mm}$ ). For each of the 15 targets, the geometric distance $D$ between the air-filled needle channel and the corresponding target center point was calculated to assess the targeting accuracy. Two points $P_{1}$ and $P_{2}$ along the needle channel and the center point $C$ of the target were manually defined via the image analysis tool of the MR system (Syngo). $D$ was calculated from the geometric distance between the straight line defined by $P_{1}$ and $P_{2}$ and the target center point $C$ (Fig. 2a).

\section{Puncture of a porcine prostate at $3 \mathrm{~T}$}

The passive tracking sequence was tested in a healthy 3-monthold domestic pig during a needle puncture of the prostate. After sedation by intravenous application of ketamine and benzoediazepine, the animal was intubated. During the experiment the animal was mechanically ventilated and general anesthesia was maintained by isofluorane gas. The MR system's spine and body array coils were used for MR signal reception.

At the beginning of the procedure, the passive marker was inserted into the animal's rectum. The proximal end of the marker was attached to the same flexible holder as used for the phantom experiments. From initial $\mathrm{T}_{2}$-weighted roadmap images of the pelvis, the center of the pig's right prostatic lobe (length $\times$ width about $60 \times 25 \mathrm{~mm}^{2}$, distance from anal sphincter muscle about $190 \mathrm{~mm}$ ) was defined as the interventional target.

During targeting, the marker was slowly moved within the rectum under POCC guidance (FLASH tracking slices: TR/TE $=4.0$ / $1.9 \mathrm{~ms}$, FOV: $320 \times 320 \mathrm{~mm}^{2}$, matrix: $256 \times 256, \alpha_{\mathrm{FLASH}}=25^{\circ}$, thickness: $5.0 \mathrm{~mm}$, partial Fourier: 5/8; bSSFP targeting slice: TR/ $\mathrm{TE}=4.0 / 1.9 \mathrm{~ms}$, FOV: $280 \times 280 \mathrm{~mm}^{2}$, matrix: $256 \times 256$, $\alpha_{\text {bSSFP }}=70^{\circ}$, thickness: $5.0 \mathrm{~mm}$, partial Fourier: $5 / 8 ; \mathrm{TA}_{\text {tot }}=1.9 \mathrm{~s}$ ). Targeting was performed until the theoretical needle axis was pointing at the center of the prostatic lobe. Then, the position of the marker was set. A customized puncture needle (length: $270 \mathrm{~mm}$, diameter: $12 \mathrm{G}$; Invivo $\mathrm{GmbH}$, Schwerin Germany) was inserted through the central opening of the marker and advanced to the target. The insertion of the needle was monitored with a standard bSSFP sequence (TR/TE $=3.75 / 1.61 \mathrm{ms,}$ FOV: $350 \times 350 \mathrm{~mm}^{2}$, matrix: $256 \times 256, \alpha=70^{\circ}$, thickness: $4.0 \mathrm{~mm}$, partial Fourier: $7 / 8$, resolution in phase-encoding direction: $80 \%$ ). Again, image position and orientation were copied from the final targeting image and passive tracking was disabled during needle insertion. The duration needed for targeting and needle insertion was measured.

\section{LITT procedure in peripheral porcine muscle tissue at $1.5 \mathrm{~T}$}

In a second animal experiment a LITT procedure was emulated in a healthy, 3-month-old domestic pig. An artificial LITT target volume (cross sectional size: length $\times$ width $=20 \times 10 \mathrm{~mm}^{2}$ ) was
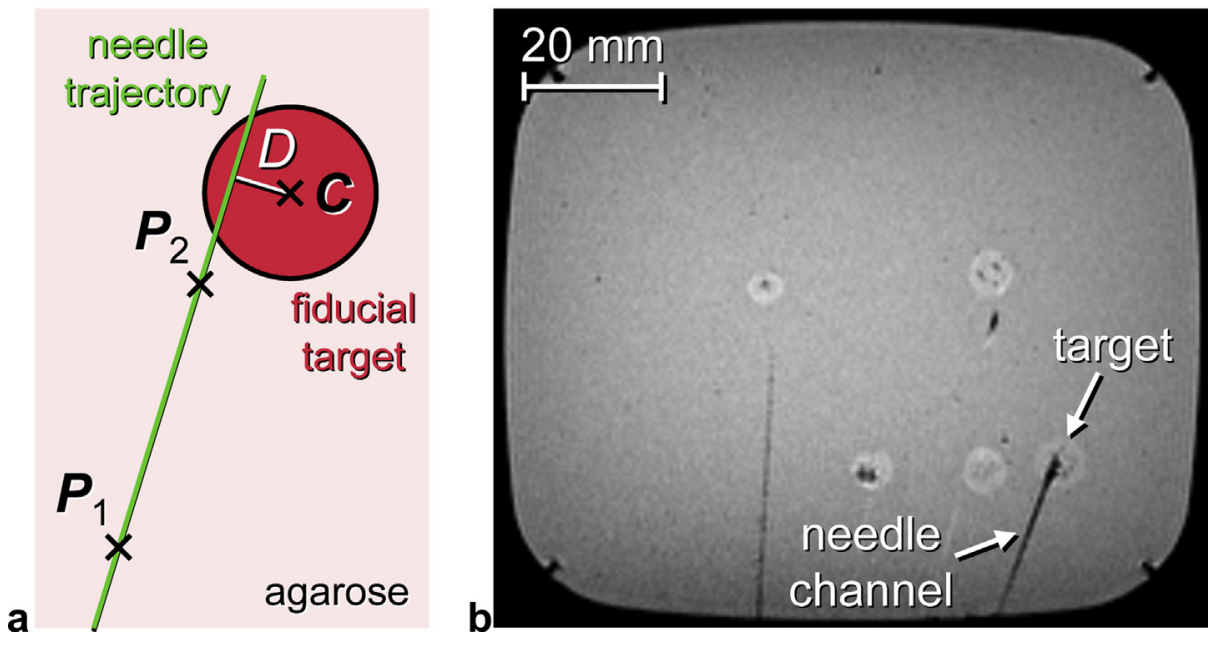

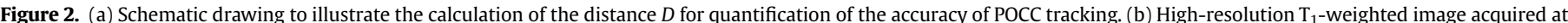

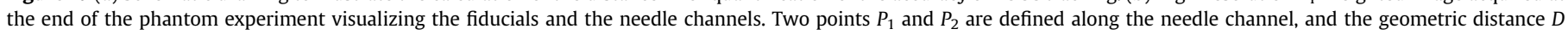
between the channel and the target center point $C$ is calculated. 
created by injecting $1.5 \mathrm{ml}$ ultrasound gel (Henry Schein Inc., Melville, New York, USA) into the muscle tissue of the pig's left hind leg at a depth of about $40 \mathrm{~mm}$ below the skin. The system's spine array coil and a loop coil were used for signal reception.

The passive marker was attached to a flexible instrument holder [13] (Invivo Germany, Schwerin, Germany) and positioned above the skin near the expected entry point of the needle. Under POCC guidance (FLASH tracking slices: TR/TE $=5.6 / 2.8 \mathrm{~ms}$, FOV: $256 \times 256 \mathrm{~mm}^{2}$, matrix: $256 \times 256, \alpha_{\mathrm{FLASH}}=35^{\circ}$, partial Fourier: $4 /$ 8, thickness: $5.0 \mathrm{~mm}$; bSSFP targeting slice: TR/TE $=5.6 / 2.8 \mathrm{~ms}$, FOV: $256 \times 256 \mathrm{~mm}^{2}$, matrix: $256 \times 256, \alpha_{\mathrm{bSSFP}}=70^{\circ}$, partial Fourier: $4 / 8$, slice thickness: $5.0 \mathrm{~mm}$; $\mathrm{TA}_{\text {tot }}=1.7 \mathrm{~s}$ ) the cylinder's symmetry axis was aligned with the center of the artificial target volume. Afterwards, the marker position and orientation were fixed by locking the holder's setscrew.

An MR-compatible puncture needle (length: $240 \mathrm{~mm}$, diameter: $18 \mathrm{G}$; Somatex GmbH, Teltow, Germany) was advanced into the target under guidance with a bSSFP sequence $(\mathrm{TR} / \mathrm{TE}=5.6 / 2.8 \mathrm{~ms}$, FOV: $256 \times 256 \mathrm{~mm}^{2}$, matrix: $256 \times 256, \alpha=70^{\circ}$, partial Fourier: 4 / 8, slice thickness: $5.0 \mathrm{~mm}$ ). Subsequently, a water-cooled LITT applicator (Somatex GmbH, Teltow, Germany) was inserted. Prior to this final step, the marker was withdrawn as the LITT applicator did not fit through the marker's central opening. The target volume was irradiated with infrared laser light $\left(\Delta t_{\text {Laser }}=90 \mathrm{~s}, P_{\text {Laser }}=20 \mathrm{~W}\right.$; Nd:YAG laser, $1.06 \mu \mathrm{m}$; mediLas $4060 \mathrm{~N}$, Dornier MedTech, Wessling, Germany). Temperature elevation was assessed via MR thermometry (segmented echo planar imaging (EPI) sequence, 3 parallel slices, TR/TE $=144 / 15 \mathrm{~ms}$, FOV: $280 \times 280 \mathrm{~mm}^{2}$, matrix: $128 \times 128$, EPI factor: 7, slice thickness $=3.0 \mathrm{~mm}$ ) based on the shift of proton resonance frequency (PRF) [14]. Treatment progress was monitored online [15] by calculation of the temperature maps and the cumulative equivalent minutes (CEM) thermal dose [16]. PostLITT $\mathrm{T}_{2}$-weighted images were acquired to estimate the size of the thermally induced lesion.

\section{Results}

\section{Accuracy evaluation in phantom at $3 T$}

The tracking and targeting images of the POCC sequence provided a sufficient image quality to identify the marker (mean marker SNR in tracking images about 60) and the fiducials (cf. Fig. 1b). All 15 targets were successfully penetrated within a mean duration for targeting and needle insertion of $2.4 \pm 1.0 \mathrm{~min}$. In the high-resolution post-intervention data set the fiducials were clearly visible (mean SNR about 80, cf. Fig. 2b). A mean tracking accuracy of $D=1.5 \pm 0.9 \mathrm{~mm}$ was measured.

\section{Puncture of a porcine prostate at $3 \mathrm{~T}$}

In the prostate experiment, the marker could be clearly distinguished from the surrounding background signal of the animal's rectum in the FLASH tracking slices (Fig. 3a and b). A marker/ background SNR of about $\mathrm{SNR}_{\text {marker }}=65 / \mathrm{SNR}_{\text {back }}=15-20$ was observed, leading to a marker-to-background signal ratio of MBR $=3-4$. For the bSSFP targeting images (Fig. 3c) a mean SNR $_{\text {bSSFP }}$ of about $30-40$ was seen. The real-time bSSFP images acquired during needle insertion provided an SNR of about 35 which was sufficient to accurately advance the needle into the center of the prostatic lobe. The final position of needle tip was confirmed by acquiring two orthogonal images with the image center positions located at a distance of about $105 \mathrm{~mm}$ from the end of the marker (Fig. 4). During the targeting procedure, the animal had to be repositioned several times due to the specific abdominal anatomy of the pig requiring multiple re-starts of the targeting maneuver (see Discussion). The entire targeting process together with the final insertion of the needle could be performed within $15 \mathrm{~min}$.
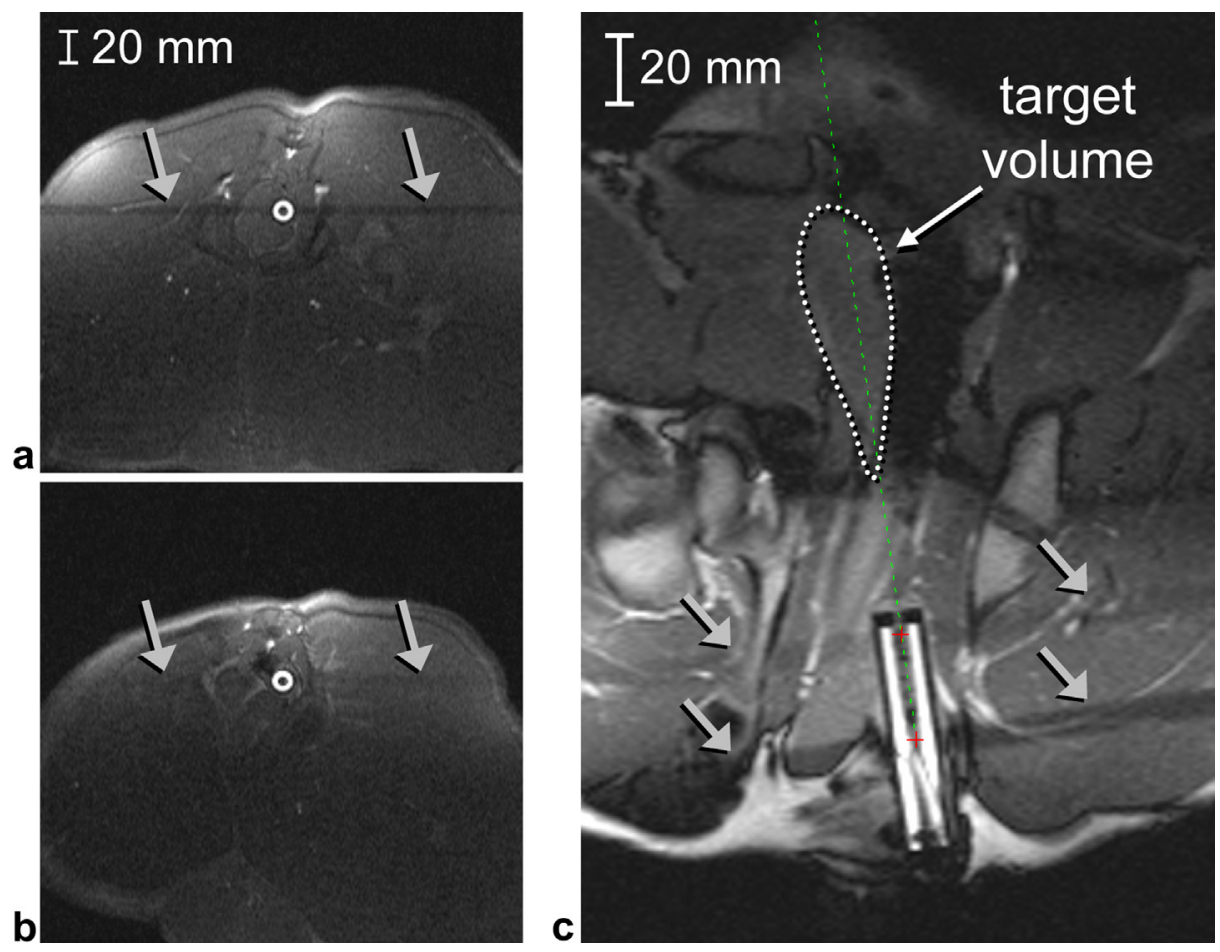

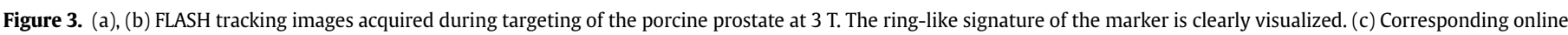

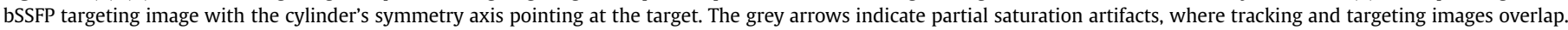



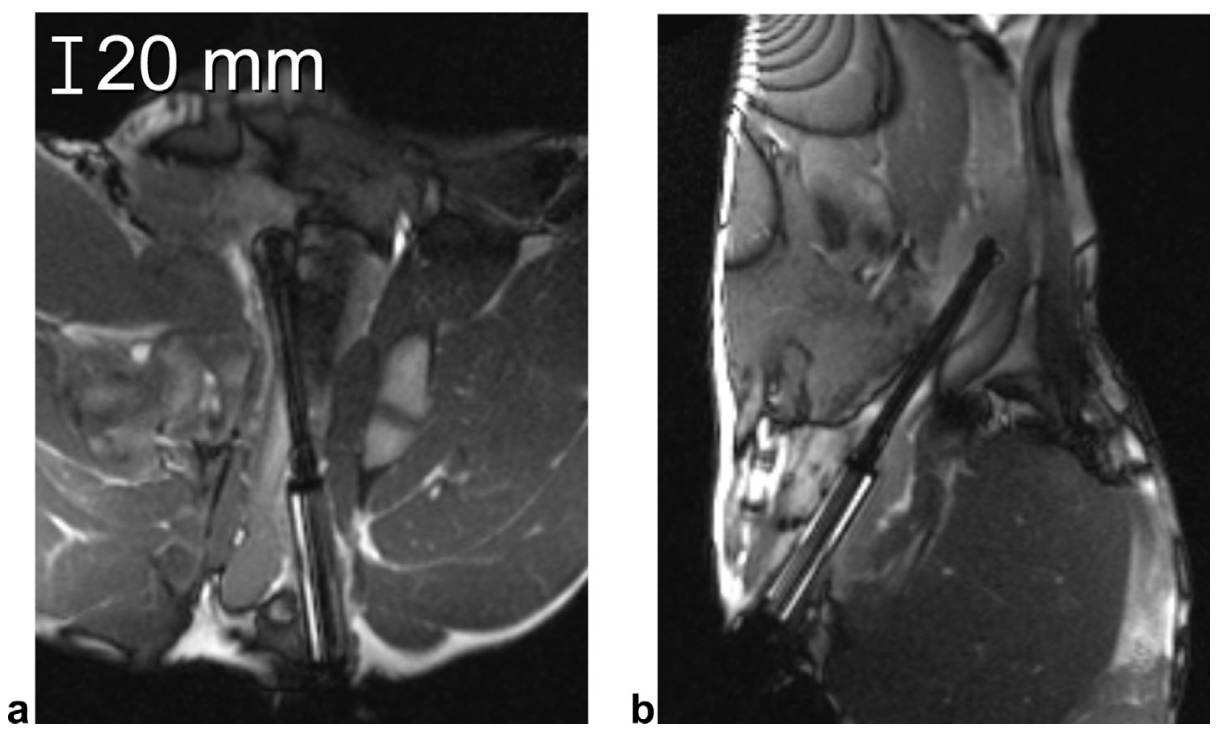

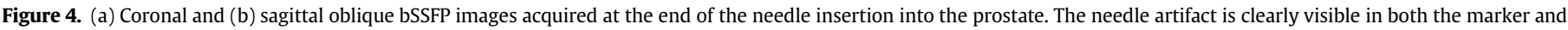

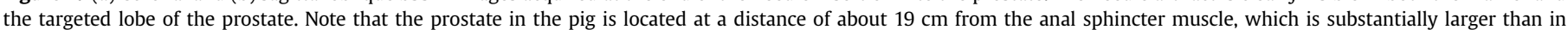
humans.

\section{LITT procedure in peripheral porcine muscle tissue at $1.5 \mathrm{~T}$}

During targeting both the passive marker and the artificial target could be clearly visualized with the POCC sequence (mean marker SNR in tracking images: $S_{N R}$ marker $=40$, mean SNR of bSSFP targeting images: SNR $_{\mathrm{bSSFP}}=20$ ). Figure 5 a shows one of the initial targeting images, Fig. 5b presents one of the bSSFP images acquired at the end of the needle insertion. The duration for targeting and needle insertion amounted to about $5.5 \mathrm{~min}$. During the exchange of the instruments (needle, LITT applicator) no displacement of the flexible instrument holder was observed. In the PRF data a maximal temperature increase of about $35 \mathrm{~K}$ was seen. Assuming a threshold for the lethal thermal dose of 240 CEM, a thermal lesion with a diameter of about $20 \mathrm{~mm}$ was estimated (Fig. 5c), which could be confirmed by post-LITT $\mathrm{T}_{2}$-weighted imaging (Fig. $5 \mathrm{~d}$ ).

\section{Discussion}

In this work, a passive tracking sequence for MR-guided needle interventions was studied in phantom and animal experiments. The sequence is based on a POCC algorithm to automatically calculate the position of a passive MR marker. In general, cross correlation algorithms have been demonstrated as versatile and powerful tools for pattern recognition in medical applications [1719]. In a recent study [20], it has been shown that cross correlation matrix computations can improve the tracking results in general over direct image intensity processing techniques such as finding maximum pixel intensities or fitting of signal profiles. In our tracking approach, the POCC algorithm is performed in the k-space domain to realize an efficient and simplified computation. With a targeting accuracy of $1.5 \mathrm{~mm}$ found in the phantom measurements, the previously reported targeting accuracy [10] could be confirmed with excellent agreement although our experiment was performed in a more demanding setting that models target depths similar to clinical situations. However, a direct translation of the measured targeting accuracy to biological tissue might be limited. As the fiducial targets were embedded in agarose gel, needle deflections e.g. due to heterogeneous mechanical properties of tissue were minimized. Furthermore, the targeting accuracy was only assessed perpendicular to the needle trajectory (similar to Ref. [10]) since an exact discrimination of the final position of the needle tip within the targets (cranberries) was difficult due to distortions of the needle channels after removing the needle. Thus, the obtained accuracy results should be considered as best-case values. Nevertheless, our results indicate that accurate targeting on the basis of the POCC technique can be achieved for remote targets of $8-10 \mathrm{~mm}$ diameter.

To study the applicability of the POCC technique under realistic conditions, the sequence was tested in two animal experiments. During tracking of the marker inside of the animal's rectum a marker-to-background-signal ratio (MBR) of about 4 was seen. Even with this apparently low MBR, tracking was successful which is consistent to the previous study [10] where robust marker tracking was demonstrated for an MBR $>4$. The entire targeting procedure together with the needle insertion into the center of the right prostatic lobe could be done within $15 \mathrm{~min}$. However, the procedure was complicated due to the curved anatomy of the porcine rectum and the greater distance of the prostate from the anal sphincter muscle (about $20 \mathrm{~cm}$, cf. Fig. 4). The animal had to be repositioned several times by placing additional cushions below the animal's belly to realize a straight needle pathway without harming surrounding tissue. Consequently, several restarts of the targeting maneuver were required so that the entire targeting procedure was prolonged. In a human transrectal application with a nearly straight rectum and a shorter distance of the prostate from anal sphincter muscle of about $7-8 \mathrm{~cm}$ no timeconsuming repositioning is required, so that POCC targeting should be achievable in shorter time. Comparable to the LITT experiment, which was performed to study POCC tracking in a percutaneous setting (similar to a prototype human application of the sequence [21]), we expect targeting and needle insertion could be done within about $5 \mathrm{~min}$.

In all experiments, the image quality of tracking and targeting images was sufficient to accurately localize the passive marker and to identify the interventional target. As the targeting images are acquired with a bSSFP sequence, banding artifacts due to offresonance effects were present (in particular at $3 \mathrm{~T}$ ) which might potentially obscure the target. Several techniques have been proposed for banding artifact suppression, e.g. summation of frequency-modulated acquisitions [22,23], which could be implemented into the sequence in future developments without compromising the temporal resolution. 

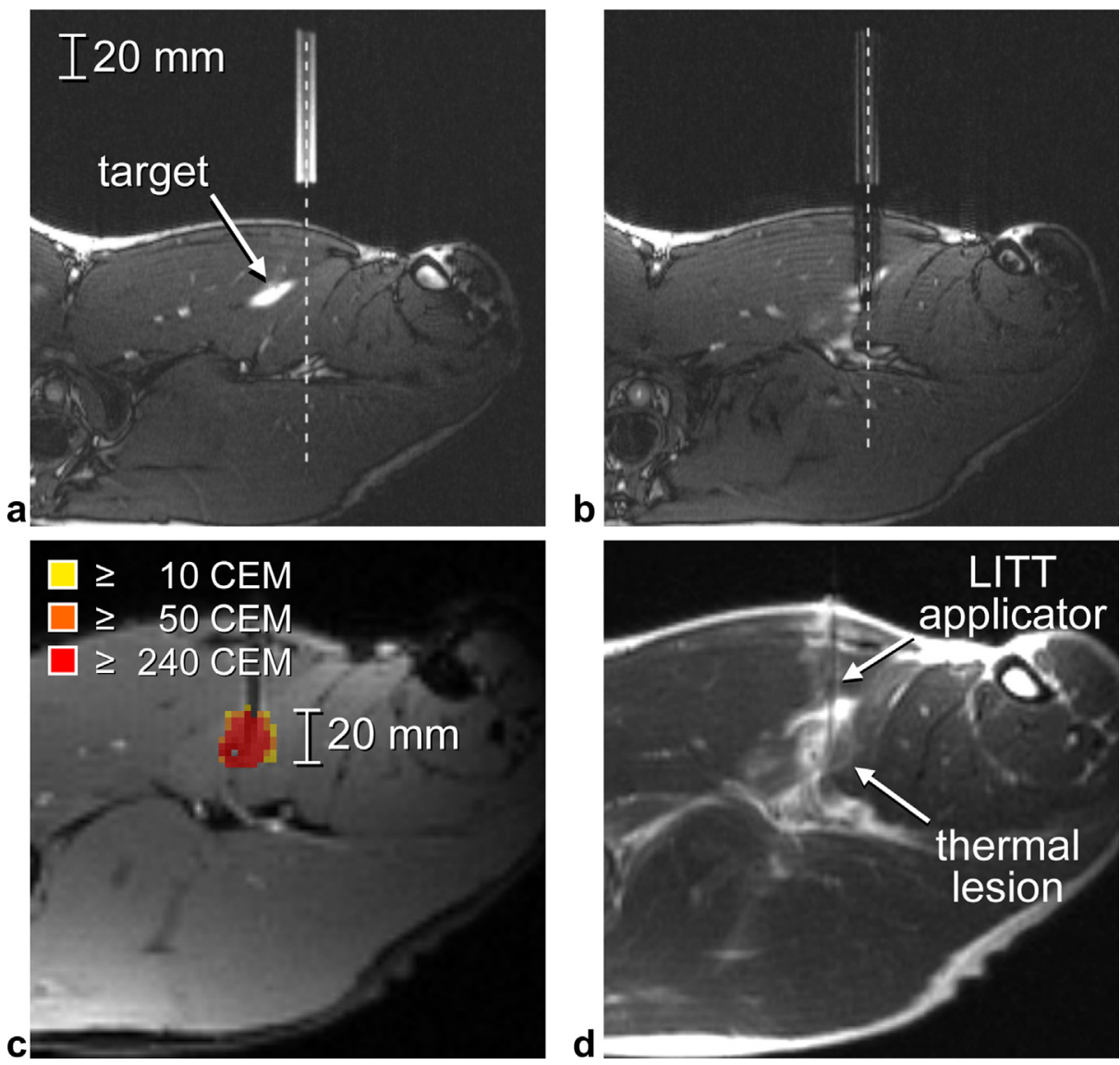

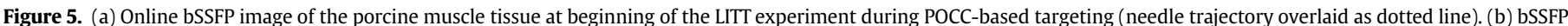

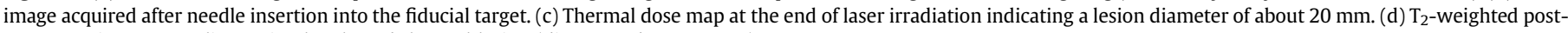
treatment image revealing a circular shaped thermal lesion (diameter about $20 \mathrm{~mm}$ ).

With a temporal resolution of $1-2 \mathrm{~s}$, the image update rate of the POCC sequence would need to be increased for the application in moving organs (e.g. LITT treatment of liver lesions). The temporal resolution could be simply improved by reducing the spatial resolution of the images which might be tolerable if larger structures are targeted. Alternatively, parallel imaging techniques could be included providing acceleration factors of 2 or more at the expense of image SNR. Nevertheless, the current image update rate of about 1-2 $\mathrm{s}$ appeared to be sufficient for applications in static organs or slowly moving targets such as the prostate.

In its current status, the POCC sequence can track the position of the passive marker only during targeting as the metallic needle can cause severe image distortions. Thus, the tracking algorithm could fail during the most critical part of the intervention, the needle insertion. Although distortions could be reduced with spin echo techniques, this would lead to longer total acquisition times. However, during needle insertion, the marker location is kept fixed, and thus, marker tracking could be disabled. Nevertheless, monitoring of the needle insertion is mandatory to detect and avoid needle bending where deflections of the needle tip up to almost $1 \mathrm{~cm}$ have been reported [24]. Without tracking, needle monitoring could be achieved at an increased temporal resolution. To exclude displacements of the marker during needle insertion in clinical routine, the marker is designed to be used with a dedicated commercial manipulation system (DynaTRIM, Invivo Corporation, Gainesville, Florida, USA). Additionally, the marker design provides an inherent guidance for the needle so that deflections are expected to be reduced as indicated by the animal experiments where nearly no needle bending was seen (cf. Figs. 4 and 5). Position and orientation of the imaging slice for monitoring of the needle pathway can be copied from the final targeting images and even optimized imaging techniques for needle visualization such as $[25,26]$ could be used.

During the experiments POCC-based tracking failed in a few occasions. For example, in the phantom experiment about 5 tracking failures occurred during targeting. These tracking failures happened due to too fast manipulations of the marker. However, such sudden movements were mainly caused by mechanical instabilities of the customized instrument holder and could be avoided by using the dedicated manipulation system. In situations of incorrect tracking, an error message was displayed on the inroom monitor and marker manipulation was immediately stopped. Then, the tracking images were manually re-positioned and passive tracking was re-started. This process took about 20$30 \mathrm{~s}$, so that the targeting process could be continued from its last position after a short delay. In general, too fast manipulations (about more than $20 \mathrm{~mm} / \mathrm{s}$ ) of the marker or strong tipping of the tracking slices against the marker's symmetry axis (angle $\geq 45^{\circ}$; [10]) lead to unwanted distortions of the ring-like marker signature within the tracking images. However, with the dedicated manipulation system rotations and translations are performed via setscrews so that sudden movements can be nearly excluded especially in transrectal procedures where the marker has to be manipulated slowly to avoid injuries e.g. of the intestinal wall.

Failures of the tracking algorithm could also occur when moving the marker in the direction of its symmetry axis. Such maneuvers are not detectable by the current implementation of the POCC tracking algorithm which is only sensitive to manipulations 
perpendicular to the symmetry axis. However, as the marker would be typically placed on the skin or close to the intestinal wall adjacent to the prostate, mainly rotations and/or lateral manipulations of the marker are required. During our animal experiments, no tracking failures due to such through-plane manipulations were observed. Nevertheless, through-plane manipulations were already addressed by modifying the marker design $[27,28]$. Unfortunately, these designs are not applicable for the use in transrectal procedures. Another concept was proposed recently using an extended POCC tracking algorithm without increasing the acquisition time [29]. This concept could be integrated into the tracking sequence for 3D passive marker tracking.

Although the POCC tracking concept together with the marker manipulation system requires additional software and hardware, no technical hardware modifications of the scanner are needed. The tracking sequence was realized with a vendor-dependent data feedback for automatic scan plane positioning. However, similar feedback functionalities are available for MR systems from other manufactures as demonstrated by recent studies on device tracking [30-32]. As a general alternative to manually performed needle interventions or focal therapies, robotic systems have been suggested for MR guidance. In previous studies with robotic systems targeting accuracies in the range of $1.3-3 \mathrm{~mm}$ have been reported [30,33-36]. Our data indicate a comparable accuracy without the need for a complex and expensive robotic technology.

POCC tracking facilitates MR-guided needle interventions because no manual and time-consuming adjustments of the imaging scan plane are required during the targeting maneuver. Image acquisitions to confirm the instrument position often prolong interventional procedures (to $1 \mathrm{~h}$ or more [3-8]). Assuming manual targeting with 10-15 manipulations of the device and interleaved conventional fast spin echo imaging (typical acquisition times about $1 \mathrm{~min}$ ) a total targeting time of about $10-15$ min would be needed. With POCC tracking, targeting could be achieved in 2$5 \mathrm{~min}$. As targeting is the most time-consuming part during a biopsy procedure, a two to three-fold reduction of the total procedure time could be achievable by using POCC tracking for MR guidance.

\section{Conclusion}

We tested a passive POCC tracking technique in phantom and animal experiments at different magnetic field strengths (1.5 T and $3 \mathrm{~T}$ ). The technical feasibility and applicability of this novel tracking approach could be demonstrated in in vivo pilot experiments. Our initial experience indicates that this tracking technique has the potential to substantially improve the entire interventional workflow. In particular, the most time-consuming part - the MRtargeting - is shortened to a few minutes by automatic scan plane positioning according to the location of the interventional device. In the next step the sequence will have to be evaluated in patients especially in MR-guided biopsies of the prostate in closebored MR systems.

\section{Acknowledgments}

The authors thank Barbara Dillenberger (Medical Physics in Radiology, German Cancer Research Center, Heidelberg, Germany) and Roland Galmbacher (University Hospital Heidelberg, Department of Anesthesiology, Heidelberg, Germany) for their support during the animal experiments.

\section{References}

[1] Weiss CR, Nour SG, Lewin JS. MR-guided biopsy: a review of current techniques and applications. J Magn Reson Imaging 2008;27(2):311-25.
[2] Bock M, Wacker FK. MR-guided intravascular interventions: techniques and applications. J Magn Reson Imaging 2008;27(2):326-38. [Epub 2008/01/26].

[3] Eliahou R, Sella T, Allweis T, Samet Y, Libson E, Sklair-Levy M. Magnetic resonance-guided interventional procedures of the breast: initial experience. Isr Med Assoc J 2009;11(5):275-9. [Epub 2009/07/30].

[4] Meeuwis C, Mann RM, Mus RDM, Winkel A, Boetes C, Barentsz JO, et al. MRIguided breast biopsy at 3T using a dedicated large core biopsy set: feasibility and initial results. Eur J Radiol, in press, http://dx.doi.org/10.1016/j.ejrad.2010. 05.001 .

[5] Anastasiadis AG, Lichy MP, Nagele U, Kuczyk MA, Merseburger AS, Hennenlotter J, et al. MRI-guided biopsy of the prostate increases diagnostic performance in men with elevated or increasing PSA levels after previous negative TRUS biopsies. Eur Urol 2006;50(4):738-49.

[6] Beyersdorff D, Winkel A, Hamm B, Lenk S, Loening SA, Taupitz M. MR imagingguided prostate biopsy with a closed MR unit at $1.5 \mathrm{~T}$ : initial results. Radiology 2005;234(2):576-81.

[7] Engelhard K, Hollenbach HP, Kiefer B, Winkel A, Goeb K, Engehausen D. Prostate biopsy in the supine position in a standard 1.5-T scanner under real time MR-imaging control using a MR-compatible endorectal biopsy device. Eur Radiol 2006;16(6):1237-43.

[8] Franiel T, Stephan C, Erbersdobler A, Dietz E, Maxeiner A, Hell N, et al. Areas suspicious for prostate cancer: MR-guided biopsy in patients with at least one transrectal US-guided biopsy with a negative findingmultiparametric MR imaging for detection and biopsy planning. Radiology 2011;259(1):162-72.

[9] Yakar D, Hambrock T, Huisman H, Hulsbergen-van de Kaa CA, van Lin E, Vergunst $\mathrm{H}$, et al. Feasibility of 3T dynamic contrast-enhanced magnetic resonance-guided biopsy in localizing local recurrence of prostate cancer after external beam radiation therapy. Invest Radiol 2010;45(3):121-5. [Epub 2010/01/13]

[10] de Oliveira A, Rauschenberg J, Beyersdorff D, Semmler W, Bock M. Automatic passive tracking of an endorectal prostate biopsy device using phase-only cross-correlation. Magn Reson Med 2008;59(5):1043-50.

[11] Chen Q-S, Defrise M, Deconinck F. Symmetric phase-only matched filtering of Fourier-Mellin transforms for image registration and recognition. IEEE Trans Pattern Anal 1994;16(12):1156-68.

[12] Foroosh H, Zerubia JB, Berthod M. Extension of phase correlation to subpixel registration. IEEE Trans Image Process 2002;11(3):188-200.

[13] Busse H, Trampel R, Gruender W, Garnov N, Fuchs J, Petersen T-O, et al. Navigation system for interventional MR image guidance in a closed-bore scanner: system setup and estimation of targeting accuracy. In: Proceedings 16th scientific meeting. Toronto, Ontario, Canada: International Society for Magnetic Resonance in Medicine; 2008 [abstract: 1214].

[14] de Poorter J, de Wagter C, de Deene Y, Thomsen C, Stahlberg F, Achten E. Noninvasive MRI thermometry with the proton resonance frequency (PRF) method: in vivo results in human muscle. Magn Reson Med 1995;33(1):7481. [Epub 1995/01/01].

[15] Maier F, Krafft AJ, Jenne JW, Semmler W, Bock M. TAM - a thermal ablation monitoring tool: in vivo evaluation. In: Dössel O, Schlegel WC, editors. World congress on medical physics and biomedical engineering, September 7-12, 2009. Munich, Germany: Springer Berlin Heidelberg; 2009. p. 247-50.

[16] Sapareto SA, Dewey WC. Thermal dose determination in cancer-therapy. Int J Radiat Oncol 1984;10(6):787-800.

[17] Zou KH, Tuncali K, Silverman SG. Correlation and simple linear regression1. Radiology 2003;227(3):617-28.

[18] Wang JZ, Reinstein LE, Hanley J, Meek AG. Investigation of a phase-only correlation technique for anatomical alignment of portal images in radiation therapy. Phys Med Biol 1996;41(6):1045-58.

[19] Paskalev K, Ma CM, Jacob R, Price R, McNeeley S, Wang L, et al. Daily target localization for prostate patients based on 3D image correlation. Phys Med Biol 2004;49(6):931-9.

[20] Rea M, McRobbie D, Elhawary H, Tse Z, Lamperth M, Young I. Sub-pixel localisation of passive micro-coil fiducial markers in interventional MRI. Magn Reson Mater Phys Biol Med 2009;22(2):71-6.

[21] Stafford RJ, Maier F, Krafft AJ, Bock M, Winkel A, Ahrar K. Phase only crosscorrelation tracking of a passive marker for MR-guided interventions. In: Proceedings 18th scientific meeting. Stockholm, Sweden: International Society for Magnetic Resonance in Medicine; 2010 [abstract: 1861].

[22] Foxall DL. Frequency-modulated steady-state free precession imaging. Magn Reson Med 2002;48(3):502-8.

[23] Zur Y, Wood ML, Neuringer LJ. Motion-insensitive, steady-state free precession imaging. Magn Reson Med 1990;16(3):444-59.

[24] Blumenfeld P, Hata N, DiMaio S, Zou K, Haker S, Fichtinger G, et al. Transperineal prostate biopsy under magnetic resonance image guidance: a needle placement accuracy study. J Magn Reson Imaging 2007;26(3):688-94.

[25] Zimmermann $H$, Mueller $S$, Gutmann B, Bardenheuer $H$, Melzer A, Umathum R, et al. Targeted-HASTE imaging with automated device tracking for MR-guided needle interventions in closed-bore MR systems. Magn Reson Med 2006;56(3):481-8.

[26] Seevinck PR, de Leeuw H, Bos C, Bakker CJG. Highly localized positive contrast of small paramagnetic objects using 3D center-out radial sampling with offresonance reception. Magn Reson Med 2011;65(1):146-56.

[27] de Oliveira A, Rauschenberg J, Beyersdorff D, Semmler W, Bock M. A new system for passive tracking of a prostate biopsy device with automatic 3D needle position estimation. In: Proceedings 16th scientific meeting. Toronto, 
Ontario, Canada: International Society for Magnetic Resonance in Medicine; 2008 [abstract: 3003].

[28] Maier F, Krafft AJ, de Oliveira A, Semmler W, Bock M. MRPen - 3D marker tracking for percutaneous interventions. In: Proceedings 17th scientific meeting. Honolulu, Hawaii, USA: International Society for Magnetic Resonance in Medicine; 2009 [abstract: 4421].

[29] Maier F, Krafft AJ, Stafford RJ, Yung JP, Dillmann R, Semmler W, et al. 3D passive marker tracking for MR-guided interventions. In: Proceedings 19th scientific meeting. Montreal, Quebec, Canada: International Society for Magnetic Resonance in Medicine; 2011 [abstract: 3749].

[30] Susil RC, Camphausen K, Choyke P, McVeigh ER, Gustafson GS, Ning H, et al. System for prostate brachytherapy and biopsy in a standard $1.5 \mathrm{~T}$ MRI scanner. Magn Reson Med 2004;52(3):683-7.

[31] Weiss S, Vernickel P, Schaeffter T, Schulz V, Gleichl B. Transmission line for improved RF safety of interventional devices. Magn Reson Med 2005;54(1): 182-9.
[32] Lederman RJ. Cardiovascular interventional magnetic resonance imaging. Circulation 2005;112(19):3009-17.

[33] Fichtinger G, Krieger A, Susil RC, Tanacs A, Whitcomb LL, Atalar E. Transrecta prostate biopsy inside closed MRI scanner with remote actuation, under realtime image guidance. Lect Notes Comput Sci 2002;2488:91-8.

[34] Krafft AJ, Jenne JW, Maier F, Stafford RJ, Huber PE, Semmler W, et al. A long arm for ultrasound: a combined robotic focused ultrasound setup for magnetic resonance-guided focused ultrasound surgery. Med Phys 2010;37(5): 2380-93.

[35] Krieger A, Susil RC, Menard C, Coleman JA, Fichtinger G, Atalar E, et al. Design of a novel MRI compatible manipulator for image guided prostate in terventions. IEEE Trans Biomed Eng 2005;52(2):306-13.

[36] Schouten MG, Ansems J, Renema WKJ, Bosboom D, Scheenen TWJ Futterer JJ. The accuracy and safety aspects of a novel robotic needle guide manipulator to perform transrectal prostate biopsies. Med Phys 2010;37(9): 4744-50. 